\title{
Smooth Extensions and Spaces of Smooth and Holomorphic Mappings
}

\author{
- preprint - \\ Christoph Wockel \\ Fachbereich Mathematik \\ Technische Universität Darmstadt \\ wockel@mathematik.tu-darmstadt.de
}

October 16, 2018

\begin{abstract}
In this paper we present another notion of a smooth manifold with corners and relate it to the commonly used concept in the literature. Afterwards we introduce complex manifolds with corners show that if $M$ is a compact (respectively complex) manifold with corners and $K$ is a smooth (respectively complex) Lie group, then $C^{\infty}(M, K)$ (respectively $C_{\mathbb{C}}^{\infty}(M, K)$ ) is a smooth (respectively complex) Lie group.
\end{abstract}

Keywords: smooth manifold with boundary; smooth manifold with corners; smooth extension; complex manifold with boundary; complex manifold with corners; infinite-dimensional Lie group; mapping group; group of smooth mappings; group of holomorphic mappings

MSC: 22E65; 58A05

\section{Introduction}

We introduce the notion of a smooth manifold with corners, which is an extension of the existing notion of smooth manifolds with corners or boundary for the finite-dimensional case (cf. Lee03, or [Mic80, Chapter 2]). The notation presented here is the appropriate notion for a treatment of mapping spaces and Whitney's extension theorem Whi34 implies that for finite-dimensional smooth manifolds our definition coincides with the one given in Lee03. We give an alternative proof of a similar statement by elementary methods from real Analysis (cf. also [KM97, Theorem 22.17] and KM97, Proposition 24.10]).

Afterwards we introduce complex manifolds with corners and show several properties of the spaces $C^{\infty}(M, K)$ and $C_{\mathrm{C}}^{\infty}(M, K)$. Eventually it turns out that these mapping spaces are smooth (respectively complex) Lie groups. This is in particular interesting since it seems to be the only way to put a complex or even smooth structure onto spaces of holomorphic mappings since very elementary results for the non-boundary case imply that compactly supported holomorphic maps are constant. With the results of this paper a topological treatment of spaces like $C_{\mathbb{C}}^{\infty}(M, K)$ for non-compact $M$ becomes possible by inductive limit methods.

\section{Notions of Differential Calculus}

In this section we present the elementary notions of differential calculus on locally convex spaces and for not necessarily open domains. 
Definition II.1. Let $E$ and $F$ be a locally convex spaces and $U \subseteq E$ be open. Then $f: U \rightarrow F$ is continuously differentiable or $C^{1}$ if it is continuous, for each $v \in E$ the differential quotient

$$
d f(x) . v:=\lim _{h \rightarrow 0} \frac{f(x+h v)-f(x)}{h}
$$

exists and if the map $d f: U \times E \rightarrow F$ is continuous. If $n>1$ we inductively define $f$ to be $C^{n}$ if it is $C^{n-1}$ and $d^{n-1} f$ is $C^{1}$ and to be $C^{\infty}$ or smooth if it is $C^{n}$ for all $n \in \mathbb{N}$. We denote the corresponding set of maps by $C^{1}(U, E), C^{n}(U, E)$ and $C^{\infty}(U, E)$. This is the notion of differentiability used in [Glö02b] and it will be the notion throughout this paper.

If $E$ and $F$ are complex vector spaces, then $f$ is called holomorphic if it is $C^{1}$ and the map $d f(x): E \rightarrow F$ is complex linear for all $x \in U$ (cf. Mil83, p.1027]).

Remark II.2. From the above definition it is clear what the notions of a smooth (respectively complex) Lie group is, i.e. a group which is a smooth (respectively complex) manifold modelled on a locally convex (complex) space such that the group operations are smooth (respectively holomorphic).

Remark II.3. (cf. Nee02, Remark 3.2]) We briefly recall the basic definitions underlying the convenient calculus from KM97. Again let $E$ and $F$ be locally convex spaces. A curve $f: \mathbb{R} \rightarrow E$ is called smooth if it is smooth in the sense of Definition 11.1 Then the $c^{\infty}$-topology on $E$ is the final topology induced from all smooth curves $f \in C^{\infty}(\mathbb{R}, E)$. If $E$ is a Fréchet space, then the $c^{\infty}$-topology is again a locally convex vector topology which coincides with the original topology KM97. Theorem 4.11]. If $U \subseteq E$ is $c^{\infty}$-open then $f: U \rightarrow F$ is said to be $C^{\infty}$ or smooth if

$$
f_{*}\left(C^{\infty}(\mathbb{R}, U)\right) \subseteq C^{\infty}(\mathbb{R}, F),
$$

e.g. if $f$ maps smooth curves to smooth curves. The chain rule Glö02a Proposition 1.15] implies that each smooth map in the sense of Definition II.1 is smooth in the convenient sense. On the other hand [KM97. Theorem 12.8] implies that on a Frèchet space a smooth map in the convenient sense is smooth in the sense of Definition 11.1 Hence for Fréchet spaces the two notions coincide.

Definition II.4. Let $E$ and $F$ be a locally convex space, and let $U \subseteq E$ be a set with dense interior. We say that a map $f: U \rightarrow F$ is $C^{1}$ if it is continuous, $f_{\text {int }}:=\left.f\right|_{\operatorname{int}(U)}$ is $C^{1}$ and the map $d\left(f_{\text {int }}\right)$ extends to a continuous map on $U \times E$, which is called the differential $d f$ of $f$. If $n>1$ we inductively define $f$ to be $C^{n}$ if if is $C^{1}$ and $d f$ is $C^{n-1}$ for $n>1$. We say that $f$ is $C^{\infty}$ or smooth if $f$ is $C^{n}$ for all $n \in \mathbb{N}_{0}$.

Remark II.5. Since $\operatorname{int}\left(U \times E^{2^{n}-1}\right)=\operatorname{int}(U) \times E^{2^{n}-1}$ we have for $n=1$ that $(d f)_{\text {int }}=d\left(f_{\text {int }}\right)$ and we inductively obtain $\left(d^{n} f\right)_{\text {int }}=d^{n}\left(f_{\text {int }}\right)$. Hence the higher differentials $d^{n} f$ are defined to be the continuous extensions of the differentials $d^{n}\left(f_{\text {int }}\right)$ and thus we have that a map $f: U \rightarrow F$ is smooth if and only if $d^{n}\left(f_{\text {int }}\right)$ has a continuous extension $d^{n} f$ to $U \times E^{2^{n}-1}$ for all $n \in \mathbb{N}$.

Remark II.6. If $f: U \rightarrow U^{\prime}, g: U^{\prime} \rightarrow F$ with $f(\operatorname{int}(U)) \subseteq \operatorname{int}\left(U^{\prime}\right)$ are $C^{1}$, then the chain rule for locally convex spaces [Glö02a, Proposition 1.15] and $(g \circ f)_{\text {int }}=g_{\text {int }} \circ f_{\text {int }}$ imply that $g \circ f: U \rightarrow F$ is $C^{1}$ and its differential is given by $d(g \circ f)(x) \cdot v=d g(f(x)) . d f(x, v)$. In particular it follows that $g \circ f$ is smooth if $g$ and $f$ are so.

Definition II.7. (cf. Lee03 for the finite-dimensional case) Let $E$ be a locally convex space, $\lambda_{1}, \ldots, \lambda_{n}$ be continuous functionals and $E^{+}:=\bigcap_{k=1}^{n} \lambda_{k}^{-1}\left(\mathbb{R}_{0}^{+}\right)$. If $M$ is a Hausdorff space, then a collection $\left(U_{i}, \varphi_{i}\right)_{i \in I}$ of homeomorphisms $\varphi_{i}: U_{i} \rightarrow \varphi\left(U_{i}\right)$ called charts onto open subsets $\varphi_{i}\left(U_{i}\right)$ of $E^{+}$is a differential structure on $M$ of co-dimension $n$ if $\cup_{i \in I} U_{i}=M$ and for each pair of charts $\varphi_{i}$ and $\varphi_{j}$ with $U_{i} \cap U_{j} \neq \emptyset$ we have that the coordinate change

$$
\varphi_{i}\left(U_{i} \cap U_{j}\right) \ni x \mapsto \varphi_{j}\left(\varphi_{i}^{-1}(x)\right) \in \varphi_{j}\left(U_{i} \cap U_{j}\right)
$$


is smooth in the sense of Definition 1 I.4 Two differential structures are called compatible if their union is again a differential structure, a maximal differential structure with respect to compatibility is called an atlas and $M$ together with an atlas is called a smooth manifold with corners of co-dimension $n$.

Remark II.8. Note that the previous definition of a smooth manifold with corners coincides for $E=\mathbb{R}^{n}$ with the one given in Lee03 and in the case of co-dimension 1 and a Banach space $E$ with the definition of a manifold with boundary in Lan99, but our notion of smoothness differs. In both cases a map $f$ defined on a non-open subset $U \subseteq E$ is said to be smooth if for each point $x \in U$ there exists an open neighbourhood $V_{x} \subseteq E$ of $x$ and a smooth map $f_{x}$ defined on $V_{x}$ with $f=f_{x}$ on $U \cap V_{x}$. However, it will turn out that for finite-dimensional smooth manifolds with corners the two notions coincide.

Lemma II.9. If $M$ is a smooth manifold with corners modelled on the locally convex space $E$ and $\varphi_{i}$ and $\varphi_{j}$ are two charts with $U_{i} \cap U_{j} \neq \emptyset$, then $\varphi_{j} \circ \varphi_{i}^{-1}\left(\operatorname{int}\left(\varphi_{i}\left(U_{i} \cap U_{j}\right)\right)\right) \subseteq \operatorname{int}\left(\varphi_{j}\left(U_{i} \cap U_{j}\right)\right)$.

Proof. Denote by $\alpha: \varphi_{i}\left(U_{i} \cap U_{j}\right) \rightarrow \varphi_{j}\left(U_{i} \cap U_{j}\right), x \mapsto \varphi_{j}\left(\varphi_{i}^{-1}(x)\right)$ and $\beta=\alpha^{-1}$ the corresponding coordinate changes. We claim that $d \alpha(x): E \rightarrow E$ is an isomorphism if $x \in \operatorname{int}\left(\varphi_{i}\left(U_{i} \cap U_{j}\right)\right)$. Since $\beta$ maps a neighbourhood $W_{x}$ of $\alpha(x)$ into int $\left(\varphi_{i}\left(U_{i} \cap U_{j}\right)\right)$ we have $d \alpha\left(\beta\left(x^{\prime}\right)\right) .\left(d \beta\left(x^{\prime}\right) \cdot v\right)=v$ for $v \in E$ and $x^{\prime} \in \operatorname{int}\left(W_{x}\right)$ (cf. Remark [1.6). Since $x^{\prime}, v \mapsto d \alpha\left(\beta\left(x^{\prime}\right)\right) .\left(d \beta\left(x^{\prime}\right) . v\right)$ is continuous and $\operatorname{int}\left(W_{x}\right)$ is dense in $W_{x}$, we thus have that $v \mapsto d \beta(\alpha(x)) \cdot v$ is a continuous inverse for $d \alpha(x)$.

Now suppose $x \in \operatorname{int}\left(\varphi_{i}\left(U_{i} \cap U_{j}\right)\right)$ and $\alpha(x) \notin \operatorname{int}\left(\varphi_{j}\left(U_{i} \cap U_{j}\right)\right)$. Then $\lambda_{i}(\alpha(x))=0$ for some $i \in\{1, \ldots, n\}$ and thus there exists an $v \in E$ such that $\alpha(x)+t v \in \varphi_{j}\left(U_{i} \cap U_{j}\right)$ for $t \in[0,1]$ and $\alpha(x)+t v \notin \varphi_{j}\left(U_{i} \cap U_{j}\right)$ for $t \in[-1,0)$. But then $v \notin \operatorname{im}(d \alpha(x))$, contradicting the surjectivity of $d \alpha(x)$.

Remark II.10. The preceding Lemma shows that the points of int $\left(E_{+}\right)$are invariant under coordinate changes and thus the interior $\operatorname{int}(M)=\bigcup_{i \in I} \varphi_{i}^{-1}\left(\operatorname{int}\left(E_{+}\right)\right)$is an intrinsic property of $M$. We denote by $\partial M:=M \backslash \operatorname{int}(M)$ th boundary of $M$.

Definition II.11. A map $f: M \rightarrow N$ between smooth manifolds with corners is said to be $C^{n}$, respectively smooth, if $f(\operatorname{int}(M)) \subseteq \operatorname{int}(N)$ and the corresponding coordinate representation

$$
\varphi_{i}\left(U_{i} \cap f^{-1}\left(U_{j}\right)\right) \ni x \mapsto \varphi_{j}\left(f\left(\varphi_{i}^{-1}(x)\right)\right) \in \varphi_{j}\left(U_{j}\right)
$$

is $C^{n}$ respectively smooth for each pair $\varphi_{i}$ and $\varphi_{j}$ of charts on $M$ and $N$. We again denote the corresponding spaces of mappings by $C^{n}(M, N)$ and $C^{\infty}(M, N)$.

Remark II.12. For a map $f$ to be smooth it suffices to check that

$$
\varphi\left(U \cap f^{-1}(V)\right) \ni x \mapsto \varphi^{\prime}\left(f\left(\varphi^{-1}(x)\right)\right) \in \psi(V)
$$

maps $\operatorname{int}\left(\varphi\left(U \cap f^{-1}(V)\right)\right)$ into $\operatorname{int} \psi(V)$ and is smooth in the sense of Definition $\llbracket .4$ for each $m \in M$ and an arbitrary pair of charts $\varphi: U \rightarrow E^{+}$and $\psi: V \rightarrow F_{+}$around $m$ and $f(m)$ due to Lemma \.6 and Lemma Џ.9

Definition II.13. If $M$ is a smooth manifold with corners and differentiable structure $\left(U_{i}, \varphi_{i}\right)_{i \in I}$, which is modelled on the locally convex space $E$, then the tangent space in $m \in M$ is defined to be $T M_{m}:=\left(E \times I_{m}\right) / \sim$, where $I_{m}:=\left\{i \in I: m \in U_{i}\right\}$ and $(x, i) \sim\left(d\left(\varphi_{j} \circ \varphi_{i}^{-1}\right)\left(\varphi_{i}(m)\right) . x, j\right)$. The set $T M:=\cup_{m \in M}\{m\} \times T_{m} M$ is called the tangent bundle of $M$.

Remark II.14. Note that the tangent spaces $T_{m} M$ are isomorphic for all $m \in M$, including the boundary points.

Proposition II.15. The tangent bundle TM is a smooth manifold with corners and the map $\pi: T M \rightarrow M,(m,[x, i]) \mapsto m$ is smooth. 
Proof. Fix a differentiable structure $\left(U_{i}, \varphi_{i}\right)_{i \in I}$ on $M$. Then each $U_{i}$ is a smooth manifold with corners with respect to the differential structure $\left(U_{i}, \varphi_{i}\right)$ on $U_{i}$. We endow each $T U_{i}$ with the topology induced from the mappings

$$
\begin{aligned}
& \operatorname{pr}_{1}: T U_{i} \rightarrow M, \quad(m, v) \mapsto m \\
& \operatorname{pr}_{2}: T U_{i} \rightarrow E, \quad(m, v) \mapsto v,
\end{aligned}
$$

and endow $T M$ with the topology making each map $T U_{i} \hookrightarrow T M,(m, v) \mapsto(x,[v, i])$ a topological embedding. Then $\varphi_{i} \circ \operatorname{pr}_{1} \times \operatorname{pr}_{2}: T U_{i} \rightarrow \varphi\left(U_{i}\right) \times E$ defines a differential structure on $T M$ and from the very definition it follows immediately that $\pi$ is smooth.

Corollary II.16. If $M$ and $N$ are smooth manifolds with corners, then a map $f: M \rightarrow N$ is $C^{1}$ if $f(\operatorname{int}(M)) \subseteq \operatorname{int}(N), f_{\text {int }}:=\left.f\right|_{\operatorname{int}(M)}$ is $C^{1}$ and $T f_{\text {int }}: T(\operatorname{int}(M)) \rightarrow T(\operatorname{int}(N)) \subseteq T N$ extends continuously to TM. If, in addition, $f$ is $C^{n}$ for $n \geq 2$, then the map

$$
T f: T M \rightarrow T N, \quad(m,[x, i]) \mapsto\left(f(m),\left[d\left(\varphi_{j} \circ f \circ \varphi_{i}^{-1}\right)\left(\varphi_{i}(m)\right) . x, j\right]\right)
$$

is well-defined and $C^{n-1}$.

Definition II.17. If $M$ is a smooth manifold with corners, then for $n \in \mathbb{N}_{0}$ the higher tangent bundles $T^{n} M$ are the inductively defined smooth manifolds with corners $T^{0} M:=M$ and $T^{n}:=T\left(T^{n-1} M\right)$. If $N$ is a smooth manifold with corners and $f: M \rightarrow N$ is $C^{n}$, then the higher tangent maps $T^{m} f: T^{m} M \rightarrow T^{m} N$ are the inductively defined maps $T^{0} f:=f$ and $T^{m} f:=T\left(T^{m-1} f\right)$ if $1<m \leq n$.

Corollary II.18. If $M, N$ and $O$ are smooth manifolds with corners and $f: M \rightarrow N$ and $g: N \rightarrow O$ with $f(\operatorname{int}(M)) \subseteq \operatorname{int}(N)$ and $g(\operatorname{int}(N)) \subseteq \operatorname{int}(O)$ are $C^{n}$, then $f \circ g: M \rightarrow O$ is $C^{n}$ and we have $T^{m}(g \circ f)=T^{m} f \circ T^{m} g$ for all $m \leq n$.

Proposition II.19. If $M$ is a finite-dimensional paracompact smooth manifold with corners and $\left(U_{i}\right)_{i \in I}$ is an open cover of $M$, then there exists a smooth partition of unity $\left(f_{i}\right)_{i \in I}$ subordinated to this open cover.

Proof. The construction in Hir76 Theorem 2.1] actually yields smooth functions $f_{i}: U_{i} \rightarrow \mathbb{R}$ also in the sense of Definition

\section{Extensions of Smooth Maps}

This section draws on a suggestion by Helge Glöckner and was inspired by Brö92, Chapter IV]. We relate the notions introduced in Definition $\llbracket .4$ to the usual notion of differentiability on a non-open subset $U \subseteq \mathbb{R}^{n}$ (cf. Remark II.8). We will see that, at least under some mild requirements, this notion coincides with the definition given in Definition $\amalg .4$

It should be emphasised that the results of this secrtion are not new (cf. Whi34, KM97, Theorem 22.17] and [KM97 Theorem 24.10]). The remarkable thing is that it is proved by methods of elementary Analysis.

Remark III.1. If $M$ is a smooth manifold with corners, we endow $C^{\infty}(M, F)$ with the topology making the canonical map

$$
C^{\infty}(M, F) \hookrightarrow \prod_{n \in \mathbb{N}_{0}} C\left(T^{n} M, F\right)_{c}, f \mapsto d^{n} f
$$

a topological embedding (cf. Glö02b Definition 3.1]), where $d^{n} f:=p r_{2^{n}} \circ T^{n} f$ (note $\left.T^{n} F=F^{2^{n}}\right)$. This is a locally convex vector topology on $C^{\infty}(M, F)$ and it is complete whenever $C^{\infty}(\operatorname{int}(M), F)$ is complete. Hence it is a Fréchet space if $M$ is a second countable finitedimensional smooth manifold with corners and $F$ is a Fréchet space. Note that this is not immediate if one uses the notion of smoothness on $M$ from [Lee03] or [Lan99]. 
Definition III.2. We say that the two locally convex spaces $E$ and $E^{\prime}$ satisfy the Cartesian closedness principle (shortly CCP) if for each pair of open subsets $U \subseteq E$ and $U^{\prime} \subseteq E^{\prime}$ and each locally convex space $F$ we have a well defined and linear isomorphism

$$
\wedge: C^{\infty}\left(U \times U^{\prime}, F\right) \rightarrow C^{\infty}\left(U, C^{\infty}\left(U^{\prime}, F\right)\right), \quad f^{\wedge}(x)(y)=f(x, y) .
$$

Remark III.3. Since for Fréchet spaces, our notion of differentiability and the one used in the convenient setting [KM97] coincide, any two Fréchet spaces satisfy the Cartesian closedness principle due to KM97 Lemma 3.12].

Proposition III.4. If $E$ and $E^{\prime}$ satisfy the Cartesian closedness principle, $U \subseteq E, U^{\prime} \subseteq E^{\prime}$ have dense interior and $F$ is locally convex, then we have a well-defined canonical map

$$
\wedge: C^{\infty}\left(U \times U^{\prime}, F\right) \rightarrow C^{\infty}\left(U, C^{\infty}\left(U^{\prime}, F\right)\right), f^{\wedge}(x)(y)=f(x, y) .
$$

If, moreover, $E^{\prime}$ is finite-dimensional, then this map is a linear isomorphism.

Proof. First we check that $f^{\wedge}$ actually is an element of $C^{\infty}\left(U, C^{\infty}\left(U^{\prime}, F\right)\right)$. If $x \in \operatorname{int}(U)$, then $\left.f^{\wedge}(x)\right|_{\operatorname{int}\left(U^{\prime}\right)} \in C^{\infty}\left(\operatorname{int}\left(U^{\prime}\right), F\right)$ due to the Cartesian closedness principle for $\operatorname{int}(U)$ and $\operatorname{int}\left(U^{\prime}\right)$. Since $d^{n} f$ extends continuously to the boundary so does $d^{n}\left(f^{\wedge}(x)\right)$. So ${ }^{\wedge}$ defines a map from $\operatorname{int}(U)$ to $C^{\infty}\left(U^{\prime}, F\right)$, which is continuous since $(C(X \times Y, Z))^{\wedge} \subseteq C(X, C(Y, Z))$. With Remark II.5 the smoothness of $f^{\wedge}$ follows in the same way as the continuity.

It is immediate that $\wedge$ is linear and injective. If $E^{\prime}$ is finite-dimensional, then surjectivity follows directly from the the Cartesian closedness principle for $E$ and $E^{\prime}$ and $C(X \times Y, Z) \cong C(X, C(Y, Z))$.

Lemma III.5. If $E$ is a locally convex space and $\left(f_{n}\right)_{n \in \mathbb{N}_{0}}$ is a sequence in $C^{1}(\mathbb{R}, E)$ such that $\left(f_{n}^{\prime}\right)_{n \in \mathbb{N}_{0}}$ converges uniformly on compact subsets to some $\bar{f} \in C(\mathbb{R}, E)$, then $\left(f_{n}\right)$ converges to some $f \in C^{1}(\mathbb{R}, E)$ with $f^{\prime}=\bar{f}$.

Proof. This can be proved as in the case $E=\mathbb{R}$ (cf. [Brö92, Proposition IV.1.7]).

Lemma III.6. Let $F$ be a Fréchet space. If $\left(v_{n}\right)_{n \in \mathbb{N}_{0}}$ is an arbitrary sequence in $F$, then there exists an $f \in C^{\infty}(\mathbb{R}, F)$ such that $f^{(n)}(0)=v_{n}$ for all $n \in \mathbb{N}_{0}$.

Proof. (cf. Brö92 Proposition IV.4.5] for the case $F=\mathbb{R}$ ). Let $\zeta \in C^{\infty}(\mathbb{R}, \mathbb{R})$ be such that $\operatorname{supp}(\zeta) \subseteq[-1,1]$ and $\zeta(x)=1$ if $-\frac{1}{2} \leq x \leq \frac{1}{2}$ and put $\xi(x):=x \zeta(x)$. Then $\operatorname{supp}(\xi) \subseteq[-1,1]$ and $\left.\xi\right|_{\left[-\frac{1}{2}, \frac{1}{2}\right]}=\mathrm{id}_{\left[-\frac{1}{2}, \frac{1}{2}\right]}$. Since $\xi^{k}$ is compactly supported, there exists for each $n \in \mathbb{N}$ an element $M_{n, k} \in \mathbb{R}$ such that $\left|\left(\xi^{k}\right)^{(n)}(x)\right| \leq M_{n, k}$ for all $x \in \mathbb{R}$. Now let $\left(p_{m}\right)_{m \in \mathbb{N}}$ be a sequence of seminorms defining the topology on $F$ with $p_{1} \leq p_{2} \leq \ldots$. We now choose $c_{k}>1$ such that $\left|p_{k}\left(v_{k}\right) c_{k}^{-k}\left(\xi\left(c_{k} \cdot\right)^{k}\right)^{(n)}(x)\right| \leq p_{k}\left(v_{k}\right) c_{k}^{n-k} M_{n, k}<2^{-k}$ if $n<k$. Note that this is possible since there are only finitely many inequalities for each $k$. Set $f_{m}:=\sum_{k=0}^{m} v_{k}\left(c_{k}^{-1} \xi\left(c_{k} \cdot\right)\right)^{k}$. We show that $f:=\lim _{m \rightarrow \infty} f_{m}$ has the desired properties. If $\varepsilon>0$ and $l \in \mathbb{N}$ we let $m_{\varepsilon, l}>l$ be such that $2^{-m_{\varepsilon, l}}<\varepsilon$. Thus

$$
\begin{aligned}
p_{l}\left(f_{m}^{(n)}-f_{m_{\varepsilon, l}}^{(n)}\right)=p_{l}\left(\sum_{k=1+m_{\varepsilon, l}}^{m} v_{k} c_{k}^{-k}\left(\xi\left(c_{k} \cdot\right)^{k}\right)^{(n)}\right) & \\
& \leq \sum_{k=1+m_{\varepsilon, l}}^{m} p_{k}\left(v_{k}\right) c_{k}^{n-k} M_{n, k} \leq 2^{-m_{\varepsilon, l}}<\varepsilon
\end{aligned}
$$

for all $m>m_{\varepsilon, l}$ and $n<l$. It follows for $n<l$ that $f_{m}^{(n)}$ converges uniformly to some $f^{n} \in C^{\infty}(\mathbb{R}, F)$ and the preceding lemma implies $\left(f^{n-1}\right)^{\prime}=f^{n}$, whence $f^{(n)}=f^{n}$. Since $l$ was chosen arbitrarily if follows that $f$ is smooth. Since we may also interchange differentiation and the limit by the preceding lemma and since $c_{k} \xi\left(c_{k} \cdot\right)$ equals the identity on a zero neighbourhood we thus have $f^{(n)}(0)=\left(\lim _{m \rightarrow \infty} f_{m}^{(n)}\right)(0)=\lim _{m \rightarrow \infty}\left(f_{m}^{(n)}(0)\right)=v_{n}$. 
Corollary III.7. If $F$ is a Fréchet space then for each $f \in C^{\infty}([0,1], F)$ there exists a $\bar{f} \in C^{\infty}(\mathbb{R}, F)$ with $\left.\bar{f}\right|_{[0,1]}=f$.

Proof. (cf. KM97, Proposition 24.10]) For $n \in \mathbb{N}_{0}$ set $v_{n}:=f^{(n)}(0)$ and $w_{n}:=f^{(n)}(1)$. Then the preceding lemma yields $f_{-}, f_{+} \in C^{\infty}(\mathbb{R}, F)$ with $f_{-}^{(n)}(0)=v_{n}=f^{(n)}(0)$ and $f_{+}^{(n)}(0)=w_{n}=f^{(n)}(1)$. Then

$$
\bar{f}(x):= \begin{cases}f_{-}(x) & \text { if } x<0 \\ f(x) & \text { if } 0 \leq x \leq 1 \\ f_{+}(x-1) & \text { if } x>1\end{cases}
$$

defines a function on $\mathbb{R}$ which has continuous differentials of arbitrary order and hence is smooth.

Theorem III.8. If $F$ is a Fréchet space and $f \in C^{\infty}\left([0,1]^{n}, F\right)$, then there exists an $\bar{f} \in$ $C^{\infty}\left(\mathbb{R}^{n}, F\right)$ with $\left.\bar{f}\right|_{[0,1]^{n}}=f$.

Proof. First we note that the Cartesian closedness principle holds in this context due to Remark III.3 and Proposition III.4 Set $f_{0}:=f$. Using Proposition III.4 we can view $f_{0}$ as an element

$$
f_{0} \in C^{\infty}\left([0,1], C^{\infty}\left([0,1]^{n-1}, E\right)\right) .
$$

which we can extend to an element of $C^{\infty}\left(\mathbb{R}, C^{\infty}\left([0,1]^{n-1}, E\right)\right)$ by Corollary @I.7 and Remark III.1 This can again be seen as an element $f_{1} \in C^{\infty}\left(\mathbb{R} \times[0,1]^{n-1}, E\right)$. In the same manner we obtain a map

$$
f_{2} \in C^{\infty}\left(\mathbb{R}^{2} \times[0,1]^{n-2}, E\right)
$$

extending $f_{1}$ as well as $f_{0}$. Iterating this procedure for each argument results in a map $\bar{f}:=f_{n}$ which extends each $f_{i}$ and so it extends $f_{0}=f$.

Proposition III.9. If $F$ is a Fréchet space, $M$ is a finite-dimensional smooth manifold and $L \subseteq M$ has dense interior and is a smooth manifold with corners with respect to the charts obtained from the restriction of the charts of $M$ to $L$, then there exists an open subset $U \subseteq M$ with $L \subseteq U$ such that for each $f \in C^{\infty}(L, F)$ there exists a $\bar{f} \in C^{\infty}(U, F)$ with $\left.\bar{f}\right|_{L}=f$.

Proof. For each $m \in \partial L \cap L$ there exists a set $L_{m}$ which is open in $M$ and a chart $\varphi_{m}: L_{m} \rightarrow \mathbb{R}^{n}$ such that $\varphi_{m}\left(L \cap L_{m}\right) \subseteq \mathbb{R}_{+}^{n}$ and $\varphi_{m}(m) \in \partial \mathbb{R}_{+}^{n}$. Then there exists a cube

$$
C_{m}:=\left[x_{1}-\varepsilon, x_{1}+\varepsilon\right] \times \ldots \times\left[x_{n}-\varepsilon, x_{n}+\varepsilon\right] \subseteq \varphi_{m}\left(L \cap L_{m}\right),
$$

where

$$
x_{i}= \begin{cases}\varphi_{m}(m)_{i} & \text { if } \varphi_{m}(m)_{i} \neq 0 \\ \varepsilon & \text { if } \varphi_{m}(m)_{i}=0\end{cases}
$$

(actually $C_{m}$ is contained in $\mathbb{R}_{+}^{n}$ and shares the $i$-th "boundary-face" with $\mathbb{R}_{+}^{n}$ if $\varphi_{m}(m)_{i}=0$ ). Then $C$ is diffeomorphic to $[0,1]^{n}$. The diffeomorphism is defined by multiplication and addition and extends to a diffeomorphism of $\mathbb{R}^{n}$. We now set $U=\operatorname{int}(L) \cup \bigcup_{m \in \partial L \cap L} V_{m}$, $V_{m}:=\operatorname{int}\left(\varphi_{m}^{-1}\left(C_{m}\right)\right)$ and choose a partition of unity $g, h,\left(h_{m}\right)_{m \in \partial L \cap L}$ subordinated to the open cover $U \backslash L, \operatorname{int}(L),\left(V_{m}\right)_{m \in \partial L \cap L}$.

If $f \in C^{\infty}(L, M)$, then Theorem $\llbracket 11.8$ yields a smooth extension $f_{m}$ of $\left.f \circ \varphi_{m}^{-1}\right|_{C_{m}}$ and thus $\bar{f}_{m}:=\left.f_{m} \circ \varphi\right|_{V_{m}}$ is smooth and extends $f$. We now set

$$
\bar{f}(x):=h(x) f(x)+\sum_{m \in \partial L} h_{m}(x) f_{m}(x),
$$

where we extend $f$ and $f_{m}$ by zero if not defined. Since $h$ (respectively $h_{m}$ ) vanishes on a neighbourhood of each point in $\partial L \cap L$ (respectively $\partial V_{m}$ ), this function is smooth and since $\left.f_{m}\right|_{V_{m} \cap L}=\left.f\right|_{V_{m} \cap L}$ for all $m \in \partial L$ it also extends $f$. 
Corollary III.10. If $U \subseteq\left(\mathbb{R}^{n}\right)^{+}$is open, $F$ a Fréchet space and $f: U \rightarrow F$ is smooth in the sense of Definition III.4 then there exists an open subset $\widetilde{U} \subseteq \mathbb{R}^{n}$, with $U \subseteq \widetilde{U}$, such that for each $f \in C^{\infty}(U, F)$ there exists a $\widetilde{f} \in C^{\infty}(\widetilde{U}, F)$ with $\left.\widetilde{f}\right|_{U}=f$.

\section{Spaces of Mappings}

In this section we prove several results on mapping spaces like $C^{\infty}(M, K)$ or $C_{\mathbb{C}}^{\infty}(M, K)$. Since many proofs carry over from the non-boundary case, we provide here only the necessary changes to the statements and extensions to [Glö02b p.366-375].

Definition IV.1. If $E$ and $F$ are locally convex complex vector spaces, $U \subseteq E$ has dense interior, then a smooth map $f: U \rightarrow F$ is called holomorphic if $f_{\text {int }}$ is holomorphic, i.e. that $d f_{\text {int }}(x): E \rightarrow F$ is complex linear (cf. Mil83 p. 1027]). We denote the space of all holomorphic functions on $U$ by $C_{\mathbb{C}}^{\infty}(U, F)$.

Remark IV.2. Note that in the above setting $d f(x)$ is complex linear for all $x \in U$ due to the continuity of the extension of $d f_{\text {int }}$.

Definition IV.3. A smooth manifold with corners is called a complex manifold with corners if it is modelled on a complex vector space $E$ and the coordinate changes in Definition $\amalg I .7$ are holomorphic. A map smooth $f: M \rightarrow N$ between complex manifolds with corners is said to be holomorphic if and for each pair of charts on $M$ and $N$ the corresponding coordinate representation is holomorphic (cf. Definition II.11). We denote the space of holomorphic mappings from $M$ to $N$ by $C_{\mathbb{C}}^{\infty}(M, N)$.

Remark IV.4. If $M$ is a complex manifold with corners and $F$ is a locally convex complex vector space, then $C_{\mathbb{C}}^{\infty}(M, F)$ is a closed subspace of $C^{\infty}(M, F)$ since the requirement on $d f(x)$ being complex linear is a closed condition as an equational requirement on $d f(x)$ in the topology defined in Remark

Lemma IV.5. If $M$ is a finite-dimensional smooth manifold with corners and $E$ and $F$ are locally convex spaces, then there is an isomorphism $C^{\infty}(M, E \times F) \cong C^{\infty}(M, E) \times C^{\infty}(M, F)$.

Proof. The proof of [Glö02b, Lemma 3.4] carries over without changes.

Lemma IV.6. If $M$ and $N$ are finite-dimensional smooth manifolds with corners, $E$ is locally convex and $f: N \rightarrow M$ is smooth, then the map $C^{\infty}(M, E) \rightarrow C^{\infty}(N, E), \gamma \mapsto \gamma \circ f$ is continuous.

Proof. The proof of [Glö02b, Lemma 3.7] carries over without changes.

Lemma IV.7. If $M$ is a finite-dimensional smooth manifold with corners and $E$ is a locally convex space, then the map $C^{\infty}(M, E) \rightarrow C^{\infty}\left(T^{n} M, T^{n} E\right), \gamma \mapsto T^{n} \gamma$ is continuous.

Proof. The proof of Glö02b Lemma 3.8] carries over for $n=1$, where Glö02b Lemma 3.7] has to be substituted by Lemma IV.7 and [Glö02b Lemma 3.4] has to be substituted by Lemma IV.5. The assertion follows from an easy induction.

Lemma IV.8. If $X$ is a Hausdorff space, $E$ and $F$ are locally convex spaces, $U \subseteq E$ is open and $f: X \times U \rightarrow F$ is continuous, then the mapping

$$
f_{\sharp}: C(X, U)_{c} \rightarrow C(X, F)_{c}, \quad \gamma \mapsto f \circ\left(\operatorname{id}_{X}, \gamma\right)
$$

is continuous.

Proof. Since the topology of compact convergence and the compact-open topology coincide on $C(X, E)$ and $C(X, F)$ Bou89a, Theorem X.3.4.2], this is Glö02b Lemma 3.9]. 
Lemma IV.9. If $M$ is a finite-dimensional smooth manifold with corners, $E$ and $F$ are locally convex spaces, $U \subseteq E$ is open and $f: M \times U \rightarrow F$ is smooth, then the mapping

$$
f_{\sharp}: C^{\infty}(M, U) \rightarrow C^{\infty}(M, F), \quad \gamma \mapsto f \circ\left(\operatorname{id}_{M}, \gamma\right)
$$

is continuous.

Proof. For $\gamma \in C^{\infty}(M, U)$ we have

$$
T\left(f_{\sharp} \gamma\right)=T\left(f \circ\left(\operatorname{id}_{M}, \gamma\right)\right)=T f \circ T\left(\operatorname{id}_{M}, \gamma\right)=T f \circ\left(\operatorname{id}_{T M}, T \gamma\right)=(T f)_{\sharp}(T \gamma)
$$

and thus inductively

$$
\begin{aligned}
& T^{n}\left(f_{\sharp} \gamma\right)=T\left(T^{n-1}\left(f_{\sharp} \gamma\right)\right)=T\left(\left(T^{n-1} f\right)_{\sharp} T^{n-1} \gamma\right) \\
& \quad=T\left(T^{n-1} f \circ\left(\operatorname{id}_{T^{n-1} M}, T^{n-1} \gamma\right)\right)=T^{n} f \circ\left(\operatorname{id}_{T^{n} M}, T^{n} \gamma\right)=\left(T^{n} f\right)_{\sharp} T^{n} \gamma .
\end{aligned}
$$

Now we can write the map $\gamma \mapsto T^{n}\left(f_{\sharp} \gamma\right)$ as the composition of the two maps $\gamma \mapsto\left(\operatorname{id}_{T^{n} M}, T^{n} \gamma\right)$ and $\left(\operatorname{id}_{T^{n} M}, T^{n} \gamma\right) \mapsto\left(T^{n} f\right)_{\sharp} T^{n} \gamma$ which are continuous by Lemma IV.7 and IV.8 Hence $f_{\sharp}$ is continuous because a map from any topological space to $C^{\infty}(M, F)$ is continuous if all compositions with $d^{n}=\operatorname{pr}_{2^{n}} \circ T^{n}$ are continuous.

Proposition IV.10. If $M$ is a compact smooth manifold with corners, $E$ and $F$ are locally convex spaces, $U \subseteq E$ is open and $f: M \times U \rightarrow F$ is smooth, then the mapping $f_{\sharp}: C^{\infty}(M, U) \rightarrow C^{\infty}(M, F), \gamma \mapsto f \circ\left(\mathrm{id}_{M}, \gamma\right)$ is smooth. If, moreover, $E$ and $F$ are complex vector spaces and $f(m): U \rightarrow F$ is holomorphic for all $m \in M$, then $f_{\sharp}$ is holomorphic.

Proof. (cf. Nee01, Proposition III.7]) We claim that

$$
d^{n}\left(f_{\sharp}\right)=\left(d_{2}^{n} f\right)_{\sharp}
$$

holds for all $n \in \mathbb{N}_{0}$, where $d_{2}^{n} f(x, y) \cdot v:=d^{n} f(x, y) .(0, v)$. This claim immediately proves the assertion due to Lemma IV.9

To verify (11) we perform an induction on $n$. The case $n=0$ is trivial, hence assume that (11) holds for $n \in \mathbb{N}_{0}$ and take

$$
\gamma \in C^{\infty}(M, U) \times C^{\infty}(M, E)^{2^{n}-1} \cong C^{\infty}\left(M, U \times E^{2^{n}-1}\right)
$$

and

$$
\eta \in C^{\infty}(M, E)^{2^{n}} \cong C^{\infty}\left(M, E^{2^{n}}\right) .
$$

Then $\operatorname{im}(\gamma) \subseteq U \times E^{2^{n}-1}$ and $\operatorname{im}(\eta) \subseteq E^{2^{n}}$ are compact and there exists an $\varepsilon>0$ such that

$$
\operatorname{im}(\gamma)+(-\varepsilon, \varepsilon) \operatorname{im}(\eta) \subseteq U \times E^{2^{n}-1} .
$$

Hence $\gamma+h \eta \in C^{\infty}\left(M, U \times E^{2^{n}-1}\right)$ for all $h \in(-\varepsilon, \varepsilon)$ and we calculate

$$
\begin{aligned}
\left(d\left(d^{n} f_{\sharp}\right)(\gamma, \eta)\right)(x) & =\lim _{h \rightarrow 0} \frac{1}{h}\left(\left(d^{n} f_{\sharp}(\gamma+h \eta)-d^{n} f_{\sharp}(\gamma)\right)(x)\right) \\
& \stackrel{i)}{=} \lim _{h \rightarrow 0} \frac{1}{h}\left(d_{2}^{n} f(x, \gamma(x)+h \eta(x))-d_{2}^{n} f(x, \gamma(x))\right) \\
& \stackrel{i i)}{=} \lim _{h \rightarrow 0} \int_{0}^{1} d_{2}\left(\left(d_{2}^{n} f(x, \gamma(x)+t h \eta(x))\right), \eta(x)\right) d t \\
& \stackrel{i i i)}{=} \int_{0}^{1} \lim _{h \rightarrow 0} d_{2}\left(\left(d_{2}^{n} f(x, \gamma(x)+t h \eta(x))\right), \eta(x)\right) d t \\
& =d_{2}^{n+1} f(x, \gamma(x), \eta(x))=\left(d_{2}^{n+1} f\right)_{\sharp}(\gamma, \eta)(x),
\end{aligned}
$$

where $i$ ) holds by the induction hypothesis, $i i$ ) holds by the Fundamental Theorem of Calculus Glö02a, Theorem 1.5] and $i i i$ ) holds due to the differentiability of parameter-dependent Integrals (cf. GN05). The derived formula $d\left(f_{\sharp}\right)=\left(d_{2} f\right)_{\sharp}$ shows that $d\left(f_{\sharp}\right)$ is complex linear. 
Corollary IV.11. If $M$ is a compact smooth manifold with corners, $E$ and $F$ are locally convex spaces, $U \subseteq E$ are open and $f: U \rightarrow F$ is smooth (respectively holomorphic), then the push forward $f_{*}: C^{\infty}(M, U) \rightarrow C^{\infty}(M, F), \gamma \mapsto f \circ \gamma$ is a smooth (respectively holomorphic) map.

Proof. Define $\tilde{f}: M \times U \rightarrow F,(x, v) \mapsto f(x)$ and apply PropositionIV.10

Proposition IV.12. Let $G$ be a group with a smooth manifold structure on $U \subseteq G$ modelled on the locally convex space $E$. Furthermore assume that there exists $V \subseteq U$ open such that $e \in V$, $V V \subseteq U, V=V^{-1}$ and

i) $V \times V \rightarrow U,(g, h) \mapsto g h$ is smooth,

ii) $V \rightarrow V, g \mapsto g^{-1}$ is smooth,

iii) for all $g \in G$ there exists an open unit neighbourhood $W \subseteq U$ such that $g^{-1} W g \subseteq U$ and the map $W \rightarrow U, h \mapsto g^{-1} h g$ is smooth.

Then there exists a unique smooth manifold structure on $G$ such that $V$ is an open submanifold of $G$ which turns $G$ into a Lie group.

Proof. The proof of [Bou89b, Proposition III.1.9.18] carries over without changes.

Theorem IV.13. Let $M$ be a compact smooth manifold with corners, $K$ be a Lie group and let $\varphi: W \rightarrow \varphi(W) \subseteq \mathfrak{k}:=L(K)$ be a chart of $K$ around $e$ with $\varphi(e)=0$. Furthermore let $\varphi_{*}: C^{\infty}(M, W) \rightarrow C^{\infty}(M, \mathfrak{k}), \gamma \mapsto \varphi \circ \gamma$.

a) If $M$ and $K$ are smooth, then $\varphi_{*}$ induces a smooth manifold structure on $C^{\infty}(M, K)$, turning it into a smooth Lie group w.r.t. pointwise operations.

b) If $M$ is smooth and $K$ is complex, then $\varphi_{*}$ induces a complex manifold structure on $C^{\infty}(M, K)$, turning it into a complex Lie group w.r.t. pointwise operations.

c) If $M$ and $K$ are complex, then the restriction of $\varphi_{*}$ to $C_{\mathbb{C}}^{\infty}(M, W)$ induces a complex manifold structure on $C_{\mathbb{C}}^{\infty}(M, K)$, turning it into a complex Lie group w.r.t. pointwise operations, modelled on $C_{\mathbb{C}}^{\infty}(M, \mathfrak{k})$.

Proof. Using Corollary IV.11 and Proposition IV.10 the proof of the smooth case in Glö02b Section 3.2] carries over to yield a). Since Proposition IV.10 also implies the holomorhpy of the group operations, b) is now immediate. Using the same argumentation as in a) we deduce c) since $\varphi_{*}$ maps $C_{\mathbb{C}}^{\infty}(M, W)$ bijectively to $C_{\mathbb{C}}^{\infty}(M, \varphi(W))$, which is open in $C_{\mathbb{C}}^{\infty}(M, \mathfrak{k})$.

\section{Acknowledgements}

The work on this paper was financially supported by a doctoral scholarship from the Land Hessen. The author would also like thank Karl-Hermann Neeb and Helge Glöckner for giving several hints to the results of this paper.

\section{References}

[Bou89a] N. Bourbaki, General Topology, Springer-Verlag, 1989.

[Bou89b] _ Lie Groups and Lie Algebras, Springer-Verlag, 1989.

[Brö92] T. Bröcker, Analysis I, BI-Wissenschaftsverlag, 1992.

[Glö02a] H. Glöckner, Infinite-Dimensional Lie Groups without Completeness Restrictions, Geometry and Analysis on Lie Groups (A. Strasburger et al, ed.), vol. 53, Banach Center Publications, 2002, pp. 43-59. 
[Glö02b] _ Lie Group Structures on Quotient Groups and Universal Complexifications for Infinite-Dimensional Lie Groups, J. Funct. Anal. 194 (2002), 347-409.

[GN05] H. Glöckner and K.-H. Neeb, An Introduction to Infinite-Dimensional Lie Groups, book in preparation, 2005.

[Hir76] M. W. Hirsch, Differential Topology, Springer-Verlag, 1976.

[KM97] A. Kriegl and P. Michor, The Convenient Setting of Global Analysis, Math. Surveys and Monographs, vol. 53, Amer. Math. Soc., 1997.

[Lan99] S. Lang, Foundations of Differential Geometry, Graduate Texts in Mathematics, vol. 191, Springer-Verlag, 1999.

[Lee03] J. M. Lee, Introduction to Smooth Manifolds, Graduate Texts in Mathematics, vol. 218, Springer-Verlag, 2003.

[Mic80] P. Michor, Manifolds of differentiable mappings, Shiva Publishing Limited, 1980, out of print, available from http://www.mat.univie.ac.at/ michor/.

[Mil83] J. Milnor, Remarks on infinite-dimensional Lie groups, Proc. Summer school on Quantum Gravity, B. De Witt ed., 1983, pp. 1008-1057.

[Nee01] K.-H. Neeb, Infinite-dimensional Lie Groups and their Representations, Infinite Dimensional Kähler manifolds (A. Huckleberry and T. Wurzbacher, eds.), vol. 31, DMVSeminar, 2001, pp. 131-177.

[Nee02] - Central extensions of infinite-dimensional Lie groups, Ann. Inst. Fourier $\mathbf{5 2}$ (2002), 1365-1442.

[Whi34] H. Whitney, Analytic extensions of differentiable functions defined on closed subsets, Trans. AMS 36 (1934), 63-89.

Christoph Wockel

Fachbereich Mathematik

Technische Universität Darmstadt

Schlossgartenstrasse 7

D-64289 Darmstadt

Germany

wockel@mathematik.tu-darmstadt.de 